\title{
A Computability Strategy for Optimization of Multiresolution Broadcast Systems: A Layered Energy Distribution Approach
}

\author{
Martin Sehlstedt, Student Member, IEEE, and James P. LeBlanc, Senior Member, IEEE
}

\begin{abstract}
This paper investigates the possible system gain for a multiresolution broadcast system using multilayer transmission of multiresolution data by utilizing nonuniform layer transmission energies. It shows how to find the energy distribution that maximizes the system performance, measured in the form of a sum of a weighted layer values $\times$ population product (representing possible revenue for service providers).

Through the introduction of the relative population coverage function $P\left(a_{i}\right)$ it is shown for a $N$ layer system that in many cases when $P\left(a_{i}\right)$ is a concave function (equivalent to $-P\left(a_{i}\right)$ being convex) it is possible to reduce what seems to be an $N$-dimensional problem to $N$ line searches. The paper also shows how the relative population coverage function can be constructed in two ways. The first uses analytic models for signal strength and population coverage (Uniform and Rayleigh). The second uses numerical signal strength and population estimates in grid format.

The paper also includes examples to illustrate how the method works and the performance gain it provides. One of the examples uses actual grid estimates for an example transmitter located in Luleå, Sweden.
\end{abstract}

Index Terms-Broadcasting, nonhomogeneous media, optimization methods, yield optimization.

\section{INTRODUCTION}

$\mathbf{T}$ ERRESTRIAL broadcast of public television has been around for some time and many countries are in the process of replacing old analog broadcasting systems with new digital ones, such as Digital Video Broadcast (DVB) [1]. The main reason for the switch is a increased image quality and improved bandwidth efficiency [2], [3]. However, with this switch the graceful image quality degradation that came, more or less for free, with the old analog system is easy to lose. The reason is the thresholding effect of a digital system, "it either works or it does not" there is no in between. For point-to-point communication this problem may be easily solved with a rate adaptive feedback algorithm. However, for a broadcast system, this is more difficult.

The DVB specification contains a partial solution by allowing hierarchical modulation making it possible to simultaneously send two different bit streams. One with base layer quality and the other with a higher quality stream. This enables receivers located with good receiving conditions to receive the high quality

Manuscript received February 3, 2005; revised June 1, 2005. This work is part of the FP6/IST project M-Pipe and is co-funded by the European Commission.

The authors are with the Department of Computer Science and Electrical Engineering, Luleå University of Technology, SE-971 87 Luleå, Sweden (e-mail: martins@sm.luth.se).

Digital Object Identifier 10.1109/TBC.2005.857603 transmission while receivers with not so good receiving conditions can at least receive the basic quality transmission. Note however, that with only two quality levels this system does not provide the graceful degradation of the old analog system. To get that type of graceful degradation one must use multiresolution with several quality steps [4], [5]. With these streams of multiresolution source data one must decide how to distribute the transmission energy between them while still making the best use of the available energy. To simplify the analysis it is assumed that each stream is transmitted over an orthogonal broadcast channel [6].

This brings us to the main questions in this paper: "is there an optimal energy distribution for a multiresolution broadcast system and can it be found?" The focus is on the distributor's perspective where the value represents the possible intake from customer fees for each received stream. This allows for a simple objective in the form of a weighted value $\times$ population product model. Some previous publications that touch on the subject are [7]-[9] which address energy distribution between competing streams or hierarchical modulation with only two layers.

To gain some insight to the optimization problem the paper begins with analysis using two simplifying assumptions. First, a simple radio propagation model is used to predict received signal strength. Secondly, it is assumed that the population within a radius can be approximated using one of two population distributions, (Uniform or Rayleigh). Some further simplifications are that all layers require the same bit rate and target bit error probability. However, the suggested method can easily be expanded to handle source coders with different bit rates for different layers by allowing multiple transmission layers with equal weights. To allow for layers with different target bit error probability complicates the analysis since each bit error probability class would require its own population coverage function. Note that any specific channel coding effects are not taken into account, as they are viewed as a trade off between error probability and bandwidth/capacity. Clearly any actual system would need some channel code to be able to detect errors.

With these simplifications and the value $\times$ population objective it is possible to analytically find optimal energy distribution for the two population distributions. With the insights gained from the analytical case the paper continues with optimization of a broadcast system where signal strength and population data is given numerically as grid data. The goal is a computability strategy for optimization of real data. The results from the simplified models are then compared with results from the actual data. 


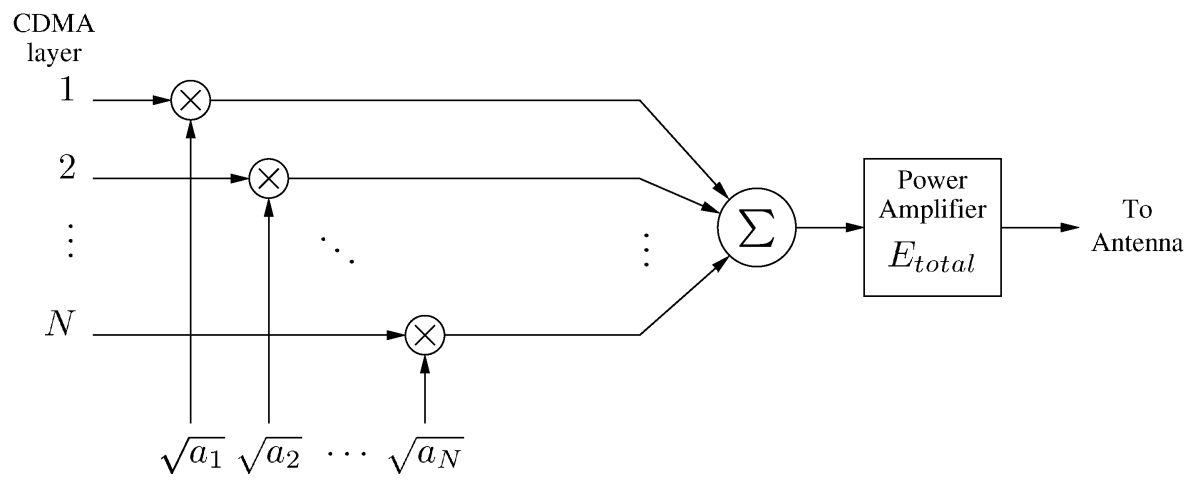

Fig. 1. $N$ Layer CDMA system where energy portions, $a_{i}$, are used to distribute the total transmitter energy, $E_{\text {total }}$, among different layers, $i$.

\section{MultiResolution BroAdCAST}

Introducing multiresolution in a broadcast system mainly affects two parts, source coding and distribution/signaling. This section gives a short introduction to the different options that are available, and motivates the selections made for this paper.

\section{A. Source Coding}

For efficient transmission of multimedia, data source coding must be utilized to reduce the amount of data bits that actually have to be transmitted. Until recently the source coding has been aimed toward achieving the highest compression ratio possible. However, with the development of cellular phones to more competent multimedia terminals and integration of the cellular networks with the Internet, WAP, and $3 \mathrm{G}$ there has been a shift in focus. The result is a more heterogeneous network with regard to terminal capabilities and connection speed. Early solutions that suggested different streams for different quality levels were soon replaced with more scalable source coders [10]. There are several ways of achieving scalability: (i) temporal, (ii) resolution, and (iii) quality to mention a few. One of the most recent video codec H.264/AVC [11]-[13] only supports temporal scalability at this point but future amendments are likely to include other types and combinations of scalability.

In this paper it is assumed that the scalability is done in layers. Consisting of one basic layer to encode the basic quality and consecutive refinement layers for higher quality, the source coder can generate a total of $N_{\max }$ layers. For simplicity it is also assumed that all layers require the same data rate and target bit error rate. One possible way to generate such data is to divide the data generated by an enhanced H.264/AVC codec for one group of pictures (GOP) into $N_{\max }$ equally sized partitions. This would make the base layer a temporal and resolution scaled version of the original and the refinement layers would then contain temporal and resolution refinement information.

\section{B. Distribution/Signaling}

With up to $N_{\max }$ concurrent data streams there are several different ways of transmitting these, some examples are hierarchical modulation or orthogonality in its various forms such as, TDMA, OFDM, or CDMA. Since the goal is to investigate how to distribute transmission energy among the different layers this paper uses QPSK signaling over orthogonal CDMA channels with only additive white Gaussian noise (AWGN) to simplify the analysis, similar to the concept used in [14]. To find the optimal energy distribution for an $N$ layer system with a total transmitter energy (denoted $E_{\text {total }}$ ) it is convenient to let $a_{i}$ denote the energy portion used for layer $i$, for $i=1, \ldots, N$, then

$$
\sum_{i=1}^{N} a_{i}=1, \quad \text { with } 0 \leq a_{i} \leq 1 \text { and } a_{i+1}<a_{i} .
$$

We have $0 \leq a_{i} \leq 1$ since the transmitted energy must be nonnegative for each layer and $a_{i+1}<a_{i}$ since in the layered coding layer $i+1$ is useful only if layer $i$ is properly received. Fig. 1 shows a simple way of using these energy portions to distribute the transmitted energy among the different layers in a $N$ layer system using orthogonal CDMA channels. This allows concurrent bit streams with just one power amplifier and transmitter antenna. This arrangement incurs the artifact of increased peak-to-average ration (PAR) for the weighted and summed modulated CDMA signals, placing increased demands on the power amplifier. However, the severity of these problems is limited if the system uses fewer than ten layers, which also limits the overall complexity of the system.

\section{OBJECTIVE AND METRIC}

Selection of an appropriate objective is indeed a subject of debate [5], as it depends on nontechnical issues (subjective performance, regulatory constraints and economic pressures). Herein it is assumed that the main objective for a broadcast system is to provide a certain service to an area, or part of the population. With a multiresolution source in the form of different layers part of the system design is to determine how many layers should be used and to partition the available transmission energy among those layers in an efficient way.

Compare with the analog terrestrial broadcast television systems where the most common metric is the signal strength at the receiver. As long as it is over a certain level the receiver will work with a minimum quality. However, this simple "above some minimum field strength" metric fails to include the scalable quality for such an analog system. Receivers closer to the transmitter will usually have better received quality than the ones located near the fringe of the covered area.

With the digital system each layer in the multiresolution transmission represents an increase in received quality and, 
as such represents some value. Therefore the objective is suggested to be a sum of weighted value $\times$ population products

$$
\mathcal{V}_{N}=\sum_{i=1}^{N} w_{i} P\left(a_{i}\right)
$$

Where the relative population coverage function, $P\left(a_{i}\right)$, represents the portion of the population that properly receives layer $i$ assuming energy portion $a_{i}$ is used for this layer. While $w_{i}$ represents the value of (or possible charge for) the increased quality the layer provides. Note that optimization is only needed if $w_{1}>w_{2}>\cdots>w_{N}$. Without loss of generality the weights can be assumed to be normalized so that $w_{1}=1$. While the actual weights depend on the actual application, we reduce the number of parameters used in the examples by introducing a one parameter model to control all weights. With the introduced weight parameter, $\beta$, the weights are calculated according to

$$
w_{i}=\beta^{i-1}, \quad \text { for } i=1, \ldots, N .
$$

Note that to fulfill the conditions for the weights $0<\beta<1$.

One of the costs associated with the use of multiresolution broadcast model presented in Section II is the multichannel circuitry and increased demand of power amplifier linearity due to the increase in peak to average power ratio. There is also another factor that may affect the objective and that is the level of required coverage. Governments may have demands that a certain part of a country's area or population be covered by a basic service level, may require additional transmitters for a multiresolution broadcast system. Both these costs can be accommodated with the simple objective but requires the inclusion of cost terms representing these additional costs of transmitters and the associated infrastructure. However, as these will vary on a case by case basis and are difficult to generalize they are not considered further in this paper.

By expressing the energy distribution in vector notation, i.e. $\mathbf{a}=\left[a_{1}, \ldots, a_{N}\right]$, finding the optimal energy distribution for a system using $N$ layers can be expressed as

$$
\mathbf{a}_{N}^{*}=\underset{\mathbf{a}}{\operatorname{argmax}} \mathcal{V}_{N} \text {. }
$$

This is a constrained $\left(\sum a_{i}=1\right.$ and $1 \geq a_{1} \geq a_{2} \geq \cdots \geq$ $\left.a_{N} \geq 0\right) N$-dimensional optimization problem that depends on the population coverage $P\left(a_{i}\right)$.

For $P\left(a_{i}\right)$ to represent the relative population which properly receives layer $i$ it must depend both on the location of the population and their ability to receive the signal at these locations. Expressed mathematically it becomes

$$
P\left(a_{i}\right)=\frac{1}{P_{0}} \int_{x} \int_{y} \operatorname{population}(x, y) \text { reception }\left(x, y, a_{i}\right) d x d y
$$

where $P_{0}$ is just a scaling factor chosen such that $P(1)=1$, population $(x, y)$ represents the population density at each location, and the ability to receive the signal is represented by

$$
\begin{aligned}
& \text { reception }\left(x, y, a_{i}\right) \\
& = \begin{cases}1 & \text { if reception of layer } i \text { possible at }(\mathrm{x}, \mathrm{y}) \\
0 & \text { otherwise. }\end{cases}
\end{aligned}
$$

Finding the truly optimal energy distribution is difficult since both the functions population $(x, y)$ and reception $\left(x, y, a_{i}\right)$ are not generally available. However, one option is to use approximations. The best available approximation for the two functions are grid data of population generated from population statistics and signal strength data in grid format generated from advanced signal propagation models. With this approximation for $P\left(a_{i}\right)$ it is possible to use a variety of numerical optimization algorithms [15]-[17]. One such example is gradient ascent. However, depending on $P\left(a_{i}\right)$ it may be possible to end up in an local (yet nonglobal) maximum if the objective function is not concave (equivalent to the negative of the objective function being convex). Another approach is to use simplifying assumptions that would allow analytical expressions for signal strength and population, as introduced in the next section.

\section{SIMPLIFYING ASSUMPTIONS}

The difficulty with the objective (2) is the unknown relative population coverage function, $P\left(a_{i}\right)$. In this section some simple analytical models for signal strength and population estimation are presented that result in analytical expressions for $P\left(a_{i}\right)$. Using these expressions the next section describes a mathematical method to find unique optimal energy distributions for these specific cases.

\section{A. Broadcast Channel}

Assuming broadcast from an omnidirectional antenna, calculation of the coverage ranges of the broadcast transmitter requires a propagation loss model. Here a simple model similar to [18] is used in favor of more recent and accurate models [19], [20] to enable mathematical analysis. However, the more advanced models may be used to generate grid data in the more complicated design example, to be presented in Section VI. With the simple model, the received signal energy is calculated as

$$
E_{\text {rec }}=E_{\text {trans }} \frac{G_{0}}{R^{\alpha}}
$$

Where $E_{\text {trans }}$ is the transmitted energy and $R$ is the distance to the transmitter in $\mathrm{km}$ while the system scaling coefficient, $G_{0}$, and the path loss exponent, $\alpha$, are constants that depend on the carrier frequency and transmitter antenna height through the parameters $A$ and $B$ described in [18],

$$
\begin{aligned}
G_{0} & =10^{-\frac{A}{10}}, \quad 94<A \leq 132 \\
\alpha & =\frac{B}{10}, \quad 29<B<36
\end{aligned}
$$

The system is assumed to use $N$ orthogonal transmission channels (for example CDMA), one for each layer, where signaling is done using QPSK over an AWGN channel. Employing such an approach, for each stream the bit error probability as a function of $R$ is

$$
p_{e}=2 Q\left(\sqrt{\frac{a_{i} E_{t o t a l} G_{0}}{\sigma^{2} R^{\alpha}}}\right),
$$



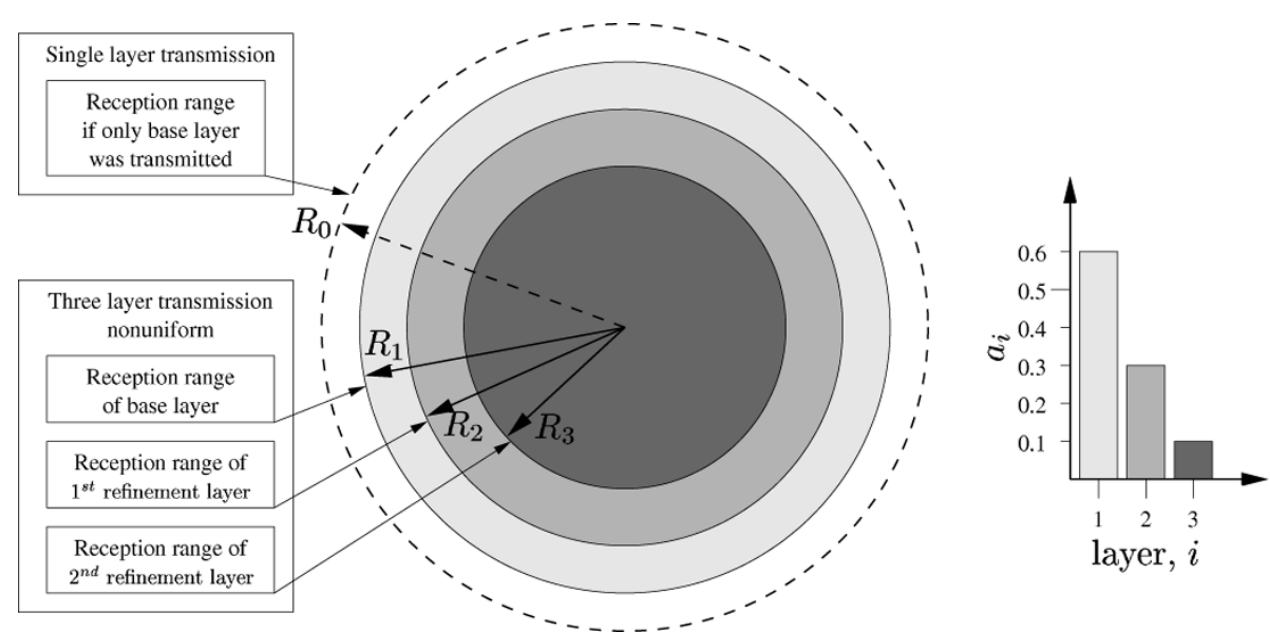

Fig. 2. Illustration of difference in coverage range for single layer $R_{0}$ and multilayer (three $R_{1}, R_{2}, R_{3}$ ) transmission using the illustrated nonuniform energy distribution for $\alpha=3.95$.

where

$$
Q(x)=\frac{1}{\sqrt{2 \pi}} \int_{x}^{\infty} e^{\frac{t^{2}}{2}} d t
$$

and $\sigma^{2}$ is the channel noise variance.

Using all energy on the base layer, (e.g. $a_{1}=1, a_{2}=$ $\left.0, \cdots, a_{N}=0\right)$, will define the maximum reachable radius, which is denoted $R_{0}$. Given some assumed target system bit error probability, $p_{e}, R_{0}$ can be expressed as

$$
R_{0}=\left(\frac{G_{0} E_{t o t a l}}{\sigma^{2} Q_{i n v}^{2}\left(\frac{p_{e}}{2}\right)}\right)^{\frac{1}{\alpha}}
$$

where $Q_{\text {inv }}(y)$ is the solution to $y=Q(x)$. Consideration of this one channel case provides, $R_{0}$, as a useful reference point which allows generalization for later optimization.

For a receiver to properly receive the information sent in a layer the received bit error probability must be less or equal to the designated system bit error probability, $p_{e}$. Thus the covered range by layer $i$ is

$$
R_{i}=\left(a_{i}\right)^{\frac{1}{\alpha}} R_{0}, \text { for } i=1, \ldots, N
$$

Fig. 2 illustrates a three layer, $N=3$, contrived example of how the different layers will have different coverage ranges if a nonuniform transmitted energy distribution is used.

\section{B. Population Distribution}

With the simple path loss model introduced in the previous section it is possible to calculate the reach of a broadcast transmission. In this section two population distributions are introduced that make it possible to estimate what part of the population is reached by the broadcast transmission. The first is a uniform model and the second is a Rayleigh model. Both were chosen since they are easily computable and have different properties.

A uniform population distribution yields that the population covered is proportional to the covered area. Under this assump- tion the relative population receiving a layer $i$ transmitted using energy portion $a_{i}$ can be written as a closed from expression

$$
P_{\text {Uniform }}\left(a_{i}\right)=\left(a_{i}\right)^{\frac{2}{\alpha}} \text {. }
$$

The second population distribution considered is Rayleigh. Here a population spread factor, $\gamma$, is used to control the population concentration around the transmitter. Valid values for the spread factor are $0<\gamma$, but already for $\gamma=1$ the concentration is almost uniform within $R_{0}$. Using Rayleigh distribution for the population receiving layer $i$ transmitted using energy portion $a_{i}$ one gets the following closed form expression for the relative population coverage function

$$
P_{\text {Rayleigh }}\left(a_{i}\right)=\frac{1-\exp \left(\frac{-\left(a_{i}\right)^{\frac{2}{\alpha}}}{2 \gamma^{2}}\right)}{1-\exp \left(\frac{1}{2 \gamma^{2}}\right)} .
$$

In the next section it is shown that with the path loss model and described population models it is possible to reduce the optimization from what seems to be an constrained $N$-dimensional optimization problem to a number of line searches.

\section{AnAlytic Results}

Possible locations for an extreme value for a constrained multivariate nonlinear function are critical points within the interior or points on constraining edges. With an objective according to (2) and the constraint $\sum a_{i}=1$ from (1) the partial derivative for the general case with $N$ layers can be written as

$$
\frac{\partial \mathcal{V}_{N}}{\partial \mathbf{a}}=\left[\begin{array}{c}
\frac{\partial \mathcal{V}_{N}}{\partial a_{1}} \\
\frac{\partial \mathcal{V}_{N}}{\partial a_{2}} \\
\vdots \\
\frac{\partial \mathcal{V}_{N}}{\partial a_{N-1}} \\
\frac{\partial \mathcal{V}_{N}}{\partial a_{N}}
\end{array}\right]=\left[\begin{array}{c}
w_{1} P^{\prime}\left(a_{1}\right)-w_{N} P^{\prime}\left(a_{N}\right) \\
w_{2} P^{\prime}\left(a_{2}\right)-w_{N} P^{\prime}\left(a_{N}\right) \\
\vdots \\
w_{N-1} P^{\prime}\left(a_{N-1}\right)-w_{N} P^{\prime}\left(a_{N}\right) \\
w_{N} P^{\prime}\left(a_{N}\right)
\end{array}\right]
$$

By solving for internal critical points (i.e. $a_{N} \neq 0$ ) requires solving the equation system $\partial \mathcal{V}_{N} / \partial \mathbf{a}=\left[\begin{array}{llll}0 & \cdots & 0 & w_{N} P^{\prime}\left(a_{N}\right)\end{array}\right]^{T}$ one gets a equation system with $N$ equations with $N$ unknowns. 
However the last unknown term, $w_{N} P^{\prime}\left(a_{N}\right)$, is present in all equations and by denoting this term $\mathcal{C}_{N}$ one gets following equation system

$$
w_{i} P^{\prime}\left(a_{i}\right)=\mathcal{C}_{N}, \quad \text { for } i=1, \ldots, N
$$

In general this equation system and the constrains in (1) allow us to find the critical points, but finding these points may require an $N$-1-dimensional search. However, if the function $P\left(a_{i}\right)$ is concave then $P^{\prime}\left(a_{i}\right)$ will be strictly decreasing and therefore have an inverse and the complexity can be reduced considerably. Let $q\left(\mathcal{C}_{N} / w_{i}\right)$ denote the general inverse for the equations in (15). With this it is possible to do a linear search over $\mathcal{C}_{N}$ for a valid energy distribution, i.e.

$$
\sum_{i=1}^{N} q\left(\frac{\mathcal{C}_{N}}{w_{i}}\right)=1
$$

Note that value for the constant $\mathcal{C}_{N}$ solving the (16) is unique, let this value be represented with the constant $\mathcal{C}_{N}^{*}$. This constant represents the energy distribution for a critical interior point, and the corresponding energy distribution is calculated using

$$
a_{i}^{*}=q\left(\frac{\mathcal{C}_{N}^{*}}{w_{i}}\right), \quad \text { for } i=1, \ldots, N
$$

This means that if the relative population coverage function $P\left(a_{i}\right)$ is concave it is possible to find the unique critical interior point for an $\mathrm{N}$ layer system using a simple line search.

Two cases for which the general inverse $q\left(\mathcal{C}_{N} / w_{i}\right)$ exists in closed analytical form are for uniform and Rayleigh populations densities when used together with the simplified broadcast transmission model in Section VII. The following subsections show how the described method works for the two cases.

\section{A. Analytical Optimization for Uniform Population Distribution}

With a uniform population distribution and the simple broadcast model the relative population coverage function takes the form of (12) with first derivative

$$
P^{\prime}\left(a_{i}\right)=\frac{2}{\alpha}\left(a_{i}\right)^{\left(\frac{2}{\alpha}-1\right)} .
$$

Using this with the results from the general optimization, (15), one obtains

$$
\mathcal{C}_{N}=w_{i} \frac{2}{\alpha}\left(a_{i}\right)^{\left(\frac{2}{\alpha}-1\right)},
$$

and as constants do not affect the actual optimization it can be simplified to

$$
\frac{\mathcal{C}_{N}^{\prime}}{w_{i}}=\left(a_{i}\right)^{\left(\frac{2}{\alpha}-1\right)} .
$$

In the general analytic results it was shown that if there exists an general inverse $q\left(\mathcal{C}_{N}^{\prime} / w_{i}\right)$ for (20) it can be used to find the optimal constant $\mathcal{C}_{N}^{\prime *}$ that solves (16) for a fixed $N$. In this case

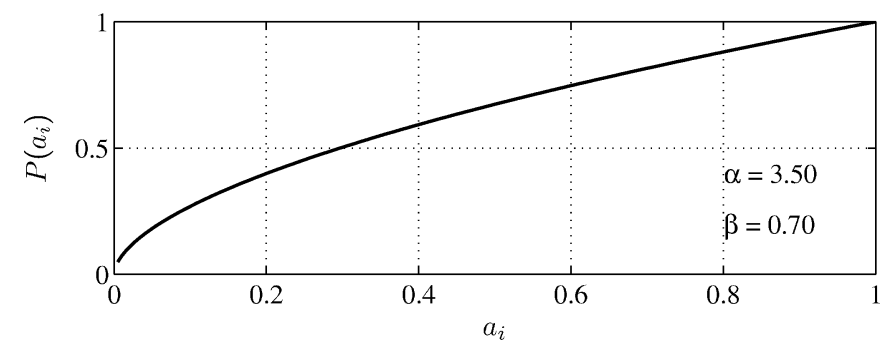

Fig. 3. Uniform optimization example, shows relative population coverage function $P\left(a_{i}\right)$ in (12).

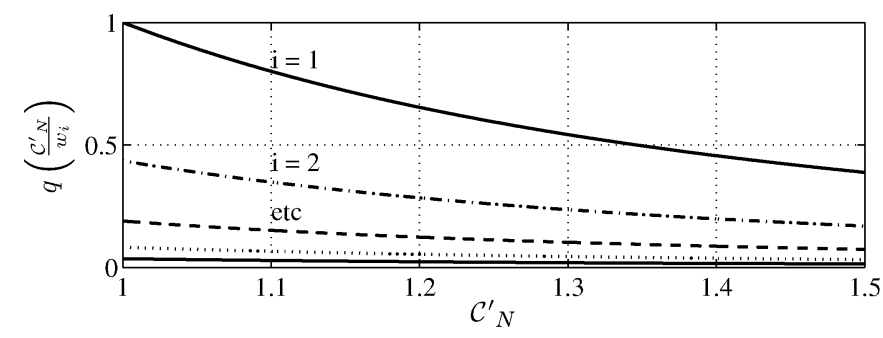

Fig. 4. Uniform optimization example, shows the effect of $w_{i}$ on the general inverse $q\left(\mathcal{C}_{N}^{\prime} / w_{i}\right)$ in (21) for some layers $i=1, \ldots, 5$.

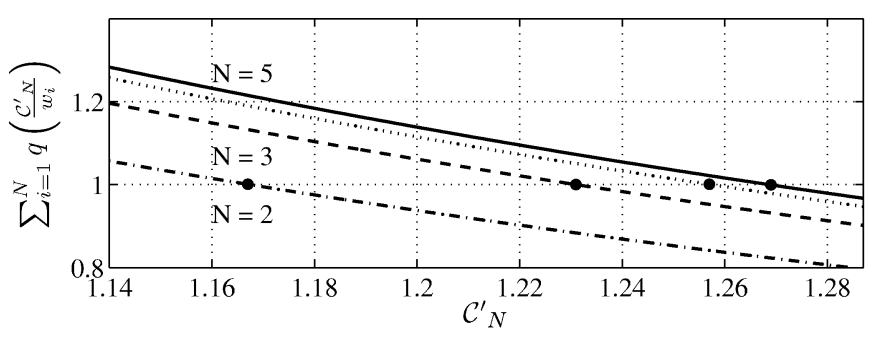

Fig. 5. Uniform optimization example, shows how the optimal constant $\mathcal{C}_{N}^{\prime *}$ is found by solving (16) for some values of $N$. The marker on each curve indicates the solution and the optimal constant $\mathcal{C}_{N}^{\prime *}$.

the general inverse $q\left(\mathcal{C}_{N}^{\prime} / w_{i}\right)$ can be written as a closed from expression

$$
q\left(\frac{\mathcal{C}_{N}^{\prime}}{w_{i}}\right)=\left(\frac{\mathcal{C}_{N}^{\prime}}{w_{i}}\right)^{\frac{\alpha}{2-\alpha}}
$$

This makes it possible to find the optimal constant $\mathcal{C}_{N}^{\prime *}$ using a single line search and through (17) it is possible to calculate the corresponding optimal energy distribution.

An illustrative example, where the following values are used for system parameters: maximum number of layers $N_{\max }=10$, objective weight parameter $\beta=0.7$, and broadcast propagation exponent $\alpha=3.5$. Fig. $3-8$ are used to illustrate how the optimization is done and to show the results. Fig. 3 shows the relative population coverage function for the uniform example, i.e. what part of the population within $R_{0}$ that is reached with layer $i$ depending on the energy portion $a_{i}$ used on that layer. Fig. 4 shows the effect of the different layer values weights $w_{i}$ in (21), the top curve is for the base layer and the curves below are for successive enhancement layers (with progressively smaller value weights $w_{i}$ ). While Fig. 5 shows how the optimal constant $\mathcal{C}_{N}^{*}$ is found by solving (16) for some different values of $\mathrm{N}$. The marker on each curve indicates the solution and the corresponding optimal constant $\mathcal{C}_{N}^{\prime *}$. The optimal constants for 1 


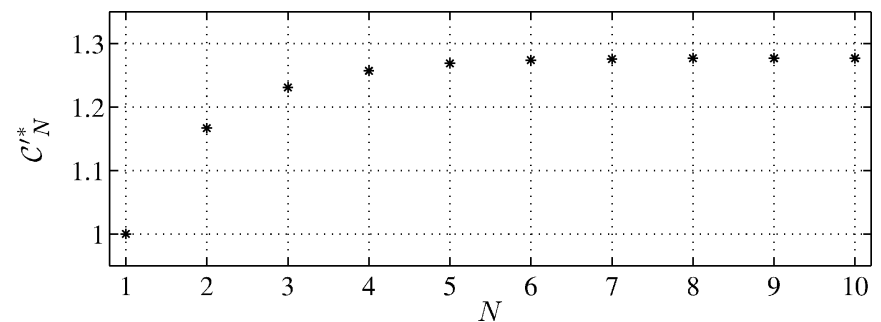

Fig. 6. Uniform optimization example, optimal constant $\mathcal{C}_{N}^{*}$ for different values of $N$.

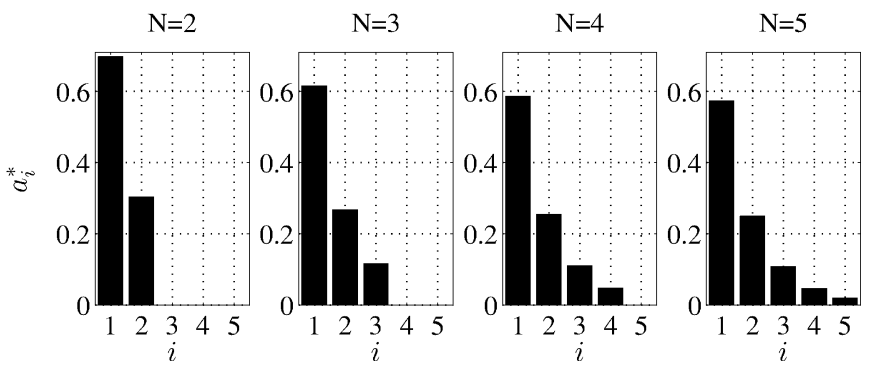

Fig. 7. Uniform optimization example, optimal energy distributions for some values of $N$.

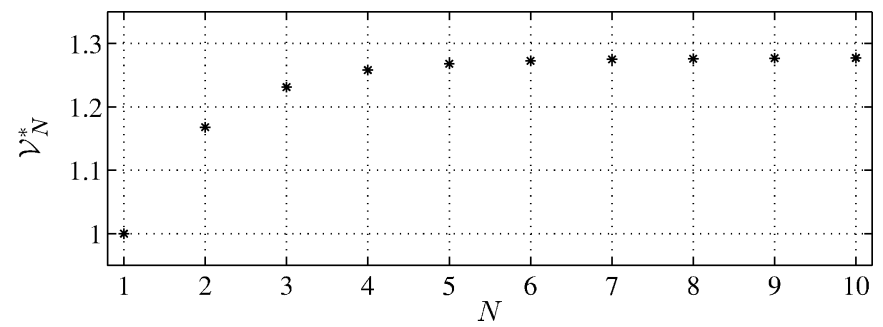

Fig. 8. Uniform optimization example, optimized objective, $\mathcal{V}_{N}^{*}$ as function of $N$.

to 10 layers are shown in Fig. 6, note that it becomes flat here above five layers. With the optimal constants $\mathcal{C}_{N}^{\prime *}$ and (21) one can use (17) to calculate the corresponding optimal energy distributions, some of which are shown in Fig. 7.

With these optimal energy distributions it is possible to evaluate the objective (2) for all possible values of $N$. These results are shown in Fig. 8 and a multilayer system shows a possible $27 \%$ gain compared to a single layer system. Note that the objective function is a monotonic increasing function of $N$, (most likely due to the fact that $\left.P^{\prime}(0)=\infty\right)$. However, with the parameters in this example $(\alpha=3.5, \beta=0.7)$ the gain becomes negligible for more than five layers.

\section{B. Analytical Optimization for Rayleigh Population Distribution}

Also for the Rayleigh population distribution, even though the relative population coverage function (13) is a bit more complicated, there is a closed form expression for the first derivative

$$
P^{\prime}\left(a_{i}\right)=\frac{1}{1-\exp \left(-\frac{1}{2 \gamma^{2}}\right)} \frac{1}{\alpha \gamma^{2}}\left(a_{i}\right)^{\left(\frac{2}{\alpha}-1\right)} \exp \left(-\frac{\left(a_{i}\right)^{\frac{2}{\alpha}}}{2 \gamma^{2}}\right) .
$$

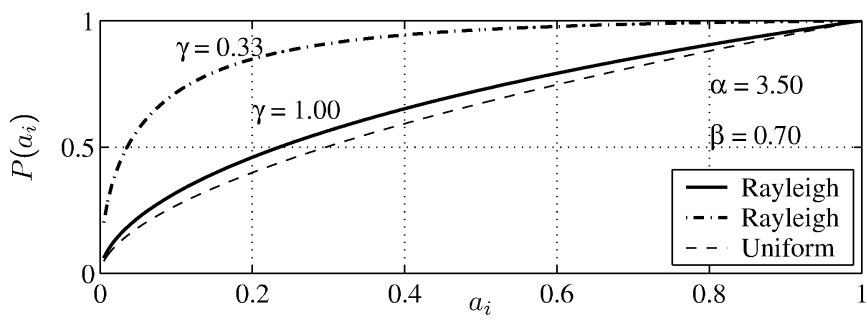

Fig. 9. Rayleigh optimization example, shows relative population coverage function $P\left(a_{i}\right)$ in (13) for the two cases. Population coverage function for the uniform case is included for comparison.

Using this with the results from the general optimization, (15), and by including all scaling in the constant $\mathcal{C}_{N}^{\prime}$ one gets

$$
\frac{\mathcal{C}_{N}^{\prime}}{w_{i}}=\left(a_{i}\right)^{\left(\frac{2}{\alpha}-1\right)} \exp \left(-\frac{\left(a_{i}\right)^{\frac{2}{\alpha}}}{2 \gamma^{2}}\right) \text {. }
$$

Also in this case the general inverse $q\left(\mathcal{C}_{N}^{\prime} / w_{i}\right)$ for (23) can be written as a closed from expression

$$
q\left(\frac{\mathcal{C}_{N}^{\prime}}{w_{i}}\right)=\left(\frac{\mathcal{C}_{N}^{\prime}}{w_{i}}\right)^{\frac{\alpha}{2-\alpha}} \exp \left(-\frac{\alpha}{2} \mathcal{W}\left(-\frac{\left(\frac{\mathcal{C}_{N}^{\prime}}{w_{i}}\right)^{\frac{2}{2-\alpha}}}{\gamma^{2}(2-\alpha)}\right)\right) .
$$

Where $\mathcal{W}(\cdot)$ is the solution to $y=x \exp (x)$, and is known as Lambert's $\mathcal{W}$-function [21], [22]. Once again a simple line search may be used to find the unique critical interior point, by solving (16).

For the Rayleigh distribution two cases are used to illustrate the procedure. Both use the same assumptions for $N_{\max }, \alpha$ and $\beta$ as for the uniform distribution. The difference between the two cases lies in the population spread factor $\gamma$ used. For case one $\gamma=1.00$ making it almost uniform and for case two $\gamma=$ 0.33 which is a more concentrated population distribution. The results of the optimizations are shown in Fig. 9-14. Fig. 9 shows the relative population coverage function for the two Rayleigh cases. For case one with population spread $\gamma=1.00$ the function is similar to the uniform case, from Fig. 3, also shown in the figure for easy comparison. Fig. 10 shows how the optimal con$\operatorname{stant} \mathcal{C}_{N}^{\prime *}$, indicated by marker on each curve, for different values of $N$ is found for the two cases by solving (16). As for the uniform case the optimal constant $\mathcal{C}_{N}^{\prime *}$ levels out for high values of $N$ the main differences is the range of the values and at which $N$ the curve becomes flat, see Fig. 11. Also in these cases the values of the optimal constant $\mathcal{C}_{N}^{\prime *}$ and (24) can be used in (17) to calculate the corresponding optimal energy distribution. some of which are shown in Fig. 12 and Fig. 13 for the two cases respectively.

With the energy distributions it is possible to evaluate the objective, (2), for all possible values of $N$. Fig. 14 shows the objective for both of the Rayleigh cases and the previous uniform example for easy comparison. Note especially the possible performance gain from using multilayer transmission. In cases where concentration of the population is high around the transmitter, as in the case where $\gamma=0.33$, a $60 \%$ gain is possible with only two layers and using more layers show a possible $150 \%$ gain. However, to achieve this high gain one has to use seven layers. 

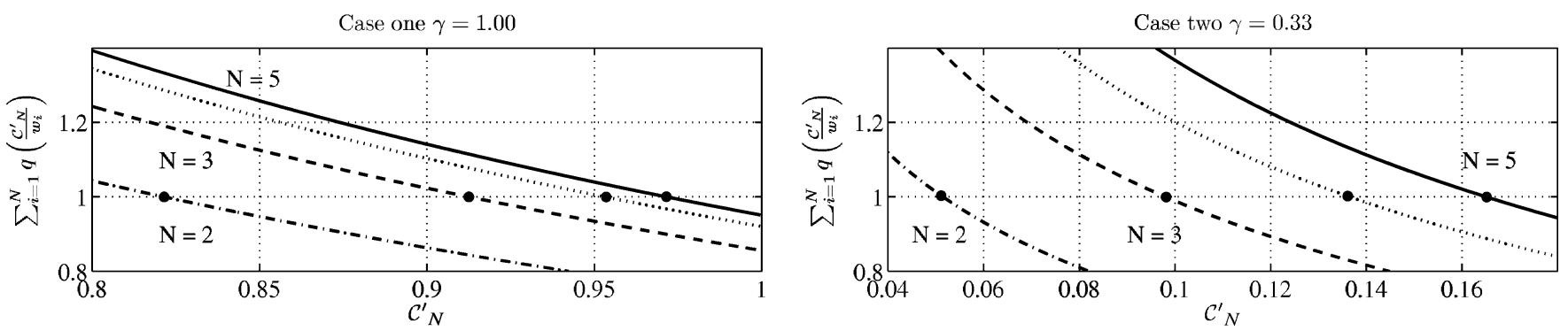

Fig. 10. Rayleigh optimization example, shows how the optimal constant $\mathcal{C}_{N}^{\prime *}$ is found for some values of $N$ by solving (16) for the two cases. The marker on each curve indicates the solution and the optimal constant $\mathcal{C}_{N}^{\prime *}$.
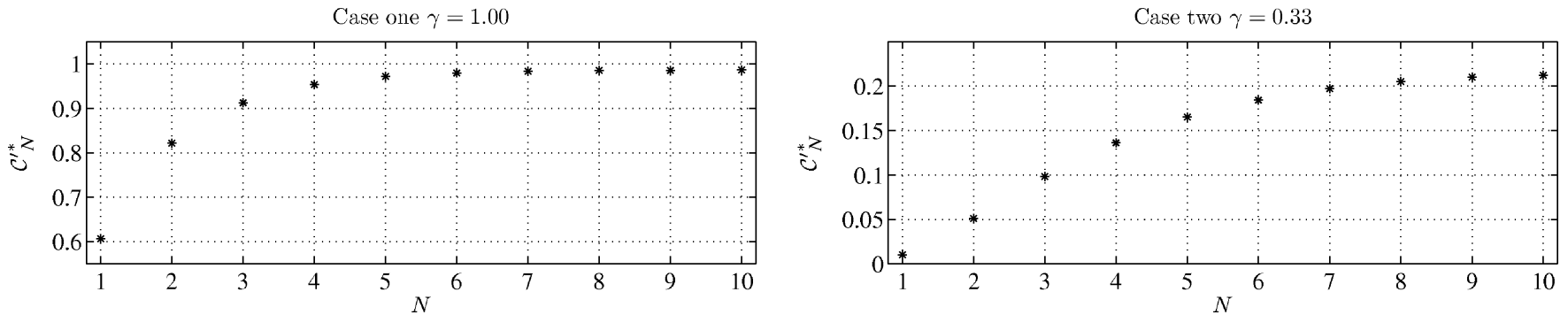

Fig. 11. Rayleigh optimization example, optimal constant $\mathcal{C}_{N}^{\prime *}$ for different $N$ for the two cases.
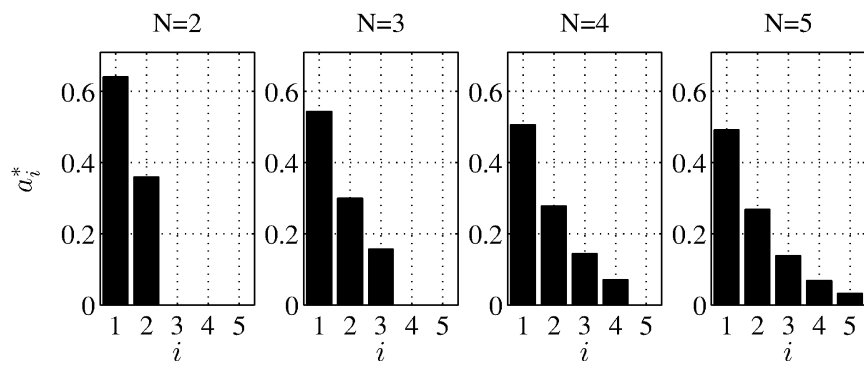

Fig. 12. Rayleigh optimization case one $\gamma=1.00$, optimal energy distribution for different values of $N$.
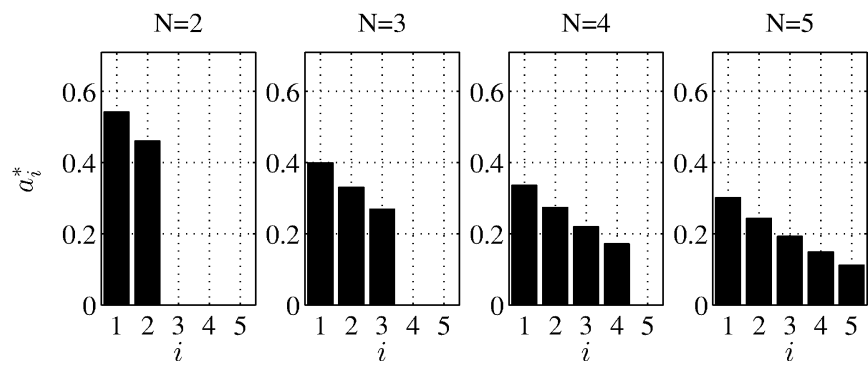

Fig. 13. Rayleigh optimization case two $\gamma=0.33$, optimal energy distribution for different values of $N$.

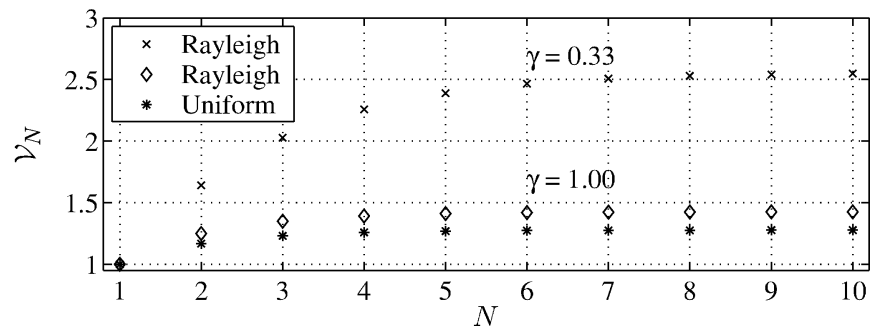

Fig. 14. Rayleigh optimization examples, optimized objective, $V_{N}^{*}$ as function of $N$.

For the first Rayleigh case, with $\gamma=1.00$, the performance is just a little better than the uniform example.

\section{GRID DATA}

Having signal strength and population data in grid form it becomes a simple matter to generate an approximation of the relative population coverage function $P\left(a_{i}\right)$. After determining a reasonable threshold value for the signal strength which allows reliable reception of the QPSK signal, a numerical approximation of (5) can be used to generate data pairs $\left(a_{i}, P\left(a_{i}\right)\right)$, corresponding to samples of the estimated function. To use this representation in optimization calculation it should be interpolated.

\section{COMPUTABILITY STRATEGY}

With the approximation of $P\left(a_{i}\right)$ an approximation of $P^{\prime}\left(a_{i}\right)$ can be found numerically and with these there are some options to find the optimal energy distribution:

Numerically: For this method a numerical search algorithm is used [15]-[17], perhaps the most common is gradient ascent.

Model approximation: It may be possible to approximate the sampled $P\left(a_{i}\right)$ using one of the simplified models. Depending on the model one needs to find an propagation exponent $\alpha$ for the broadcast propagation model and an population spread $\gamma$ for the Rayleigh distribution model if used. With these parameters one can find the corresponding unique optimum using the search for optimal constant method described below.

Search for constant: This case uses the same technique that was used to find the optimum for the uniform or Rayleigh approximations. However, it is only if the $P\left(a_{i}\right)$ approximation is concave that it is possible to find the general inverse $q\left(\mathcal{C}_{N}^{\prime} / w_{i}\right)$ used in the line search to find the optimal constant $\mathcal{C}_{N}^{\prime *}$. Note that, depending on the values of $w_{i}$, even if the general inverse $q\left(\mathcal{C}_{N}^{\prime} / w_{i}\right)$ is found it may still be impossible to find the optimal constant due to insufficient domain of $q\left(\mathcal{C}_{N}^{\prime} / w_{i}\right)$. 


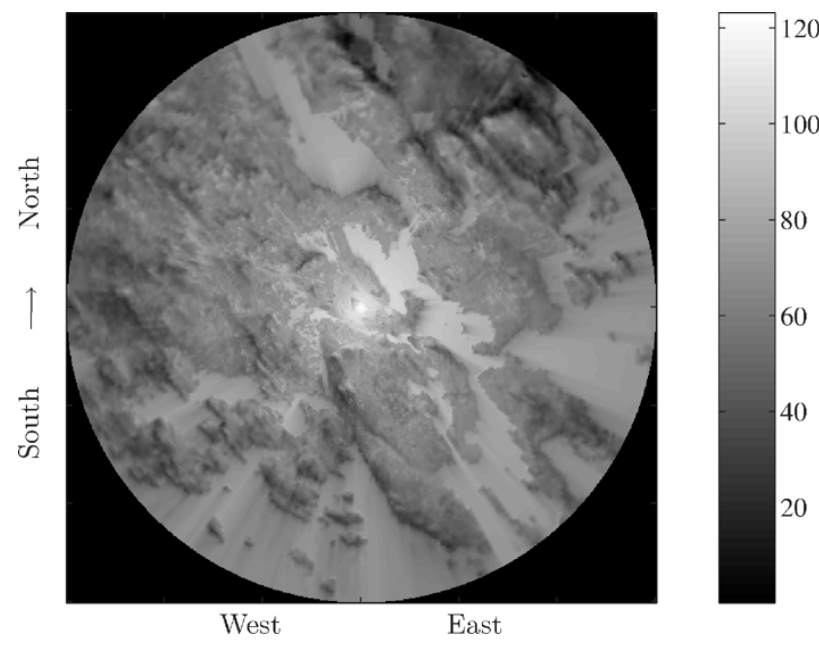

Fig. 15. The signal strength for an example transmitter on Mjölkuddsberget (located in the center of the image).

When grid data has been used to generate data pairs $\left(a_{i}, P\left(a_{i}\right)\right)$ as an approximation and the $P\left(a_{i}\right)$ is almost concave it might be possible to generate an accurate enough concave approximation by using only the data pairs that form an concave approximation [23]. However, this technique is not investigated further in this paper.

\section{Actual Data Example—City of LuleÅ, Sweden}

In this section it is shown how the optimal energy distribution can be found for an example transmitter in Luleå, a small coastal city in the north of Sweden with a population of about 70000 . The transmitter is located on Mjölkuddsberget, a small mountain close to Luleå city center, and the transmission is done at $850 \mathrm{MHz}$. The transmitter uses an omnidirectional antenna with an 72 meters effective antenna height and a receiver is assumed to have an effective antenna height of 1.5 meters. The transmitter has an effective radiated power of $1.0 \mathrm{~kW}$ and it is assumed that the receivers require a received signal strength of at least $50 \mathrm{~dB} \mu \mathrm{V} / \mathrm{m}$ for correct reception of each layer. The weighting of each layer in the objective functions is according to the one parameter weight function with $\beta=0.7$. The results from two optimization methods are compared. The first is a numerical gradient ascent the second uses a simple Rayleigh approximation.

To generate the approximation of the actual relative population coverage function, $P\left(a_{i}\right)$, grid data for both signal strength and population is needed. Both were made available through Ericsson Research $\mathrm{AB}$ and Progira Radio Communication AB. The signal strength data was generated using GiraPlan [24] which combines advanced propagation models with geographical data [25]. A map of the estimated signal strengths data with a $50 \times 50$ meter grid is shown in Fig. 15. The transmitter is located in the middle and the calculation was limited to a $15 \mathrm{~km}$ radius (hence the outer circular limit of signal strength).

The population data shown in Fig. 16 is a combination of data from population databases [26], one for population centers and one for sparsely populated areas. Both use different grids ( 250 $\times 250$ meters $)$ and $(500 \times 500$ meters $)$ respectively and were interpolated to get a $50 \times 50$ meters grid. There is a small offset within \pm 25 meters between the signal strength and population

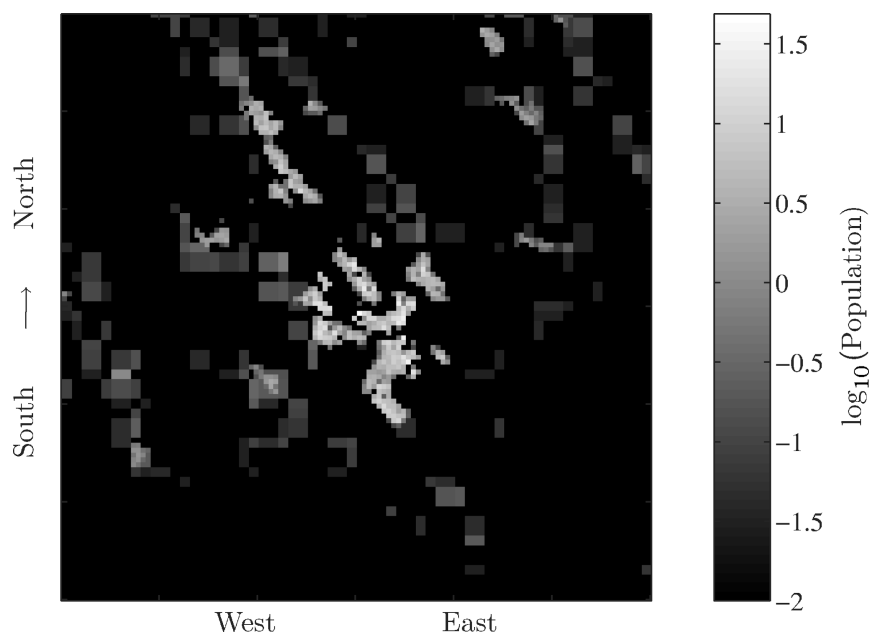

Fig. 16. Geographical distribution of population around the transmitter located on Mjölkuddsberget. Note that a logarithmic scale is used.

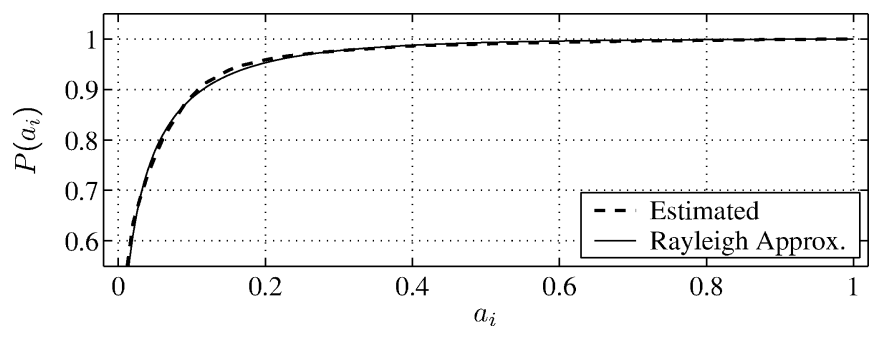

Fig. 17. Shows population coverage function $P\left(a_{i}\right)$ estimated from actual grid data and the closest Rayleigh approximation.

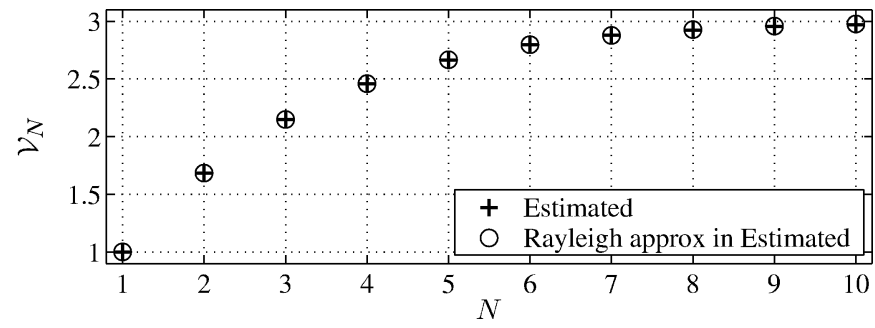

Fig. 18. Shows objective performance of optimization for both actual data and Rayleigh approximation.

grid data but the difference is less than one half grid $(50 \times 50$ meters) and is therefore believed to be negligible.

Combining the signal strength and the population data it is possible to get an estimate of the relative population coverage function $P\left(a_{i}\right)$ using the method described in Section VI, the result is shown in Fig. 17. It was not a priori expected that the estimated function would be almost concave. However, it was found to compare closely with the concave approximation using Rayleigh distribution with $\gamma=0.27$ and the path loss exponent $\alpha=3.95$ (found using curve fitting).

For the Rayleigh approximation it was shown in Section V how an unique optimal energy distribution could be found. Unfortunately the same technique can not be used in this case since the relative population coverage function $P\left(a_{i}\right)$ estimated from grid data only is guaranteed to be increasing and not necessarily concave. Therefore a gradient ascent method was used to find optimal energy distributions for some values of $N$. The resulting objective is shown in Fig. 18. Note especially the achievable performance increase. The use of two layers realizes a $60 \%$ gain 


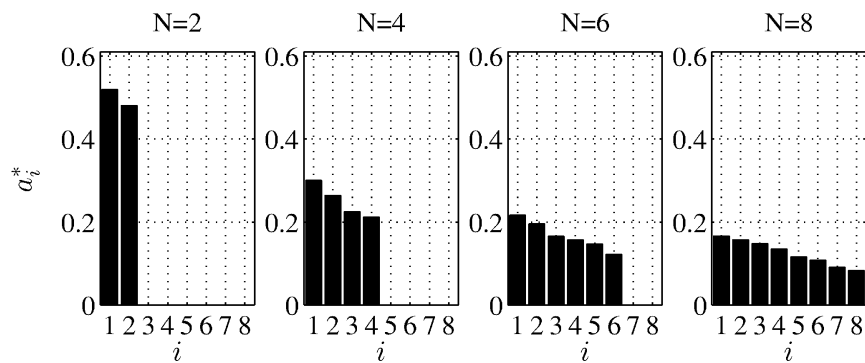

Fig. 19. Shows optimal energy distribution for actual data for some values of $N$.

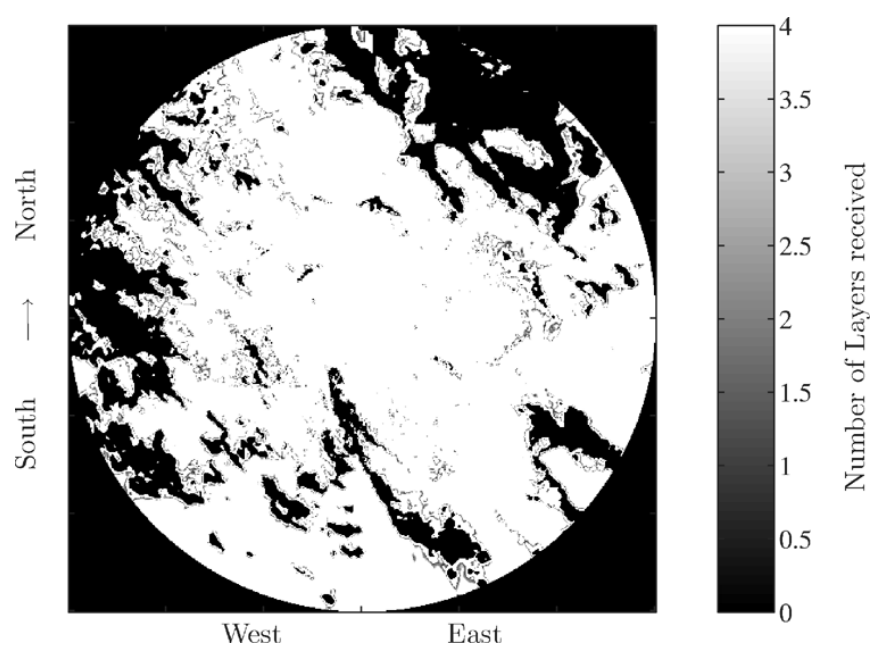

Fig. 20. Coverage map for the different layers for a four layer system using the optimal energy distribution.

compared to the one layer solution while the use of more layers can provide more gain. However, for this system there is little to be gained by use of more than seven layers which yields a $180 \%$ gain. The figure also shows the expected performance if the optimal energy distribution of the Rayleigh approximation is used in the estimated system, and as expected the difference in performance is small. The reason that the gradient optimization works so well in this case is most likely to the almost concave $P\left(a_{i}\right)$ estimate from grid data.

The resulting optimal energy distribution among the layers for the gradient search on the grid data example for some number of transmitted layers are shown in Fig. 19. While Fig. 20 shows the coverage map of the different layers for a four layer system when the optimal energy distribution is used. Note how most of the area is covered with all four layers and that it is only close to areas with no reception (black) that one may notice the different shades of gray. The explanation for this can be found in the Rayleigh approximation model, which implies that the population is concentrated close to the transmitter $(\gamma=0.27)$ and the propagation loss is large due to the high propagation loss exponent $(\alpha=3.95)$. This leads to a optimum with many layers using almost uniform energy distribution. When this energy distribution is used with the actual signal strength map, where path loss is based on more accurate models, the energy used for the four layers is enough to cover the shown area with all four layers and only locations with bad reception appear as black.

\section{CONCLUSIONS}

With increasing deployment of digital broadcast systems there is an increasing interest to maximize value of transmission and to increase network and service flexibility for such systems. One possible way is through the use of multiresolution broadcasting. Since multiresolution source coding is moving beyond research interest only and beginning to appear in international standards, this paper investigates the performance improvements available to digital multiresolution broadcast systems. In such a system it is assumed that the multiresolution source coder is used to generate source data in the form of different layers, one base layer and successive refinement layers, thereby making it desirable to maximize system performance by optimizing the energy distribution among the layers in the transmitter. With $N$ available layers this may initially appear to be a constrained $N$-dimensional problem, but it is shown here that for many cases it may be simplified to $N$ line searches.

For systems where the objective can be formulated using a weighted value $\times$ population product it was demonstrated that an optimal energy distribution can considerably increase the performance compared to a single layer system. By introducing the relative population coverage function $P\left(a_{i}\right)$, it has been shown that there exists an unique optimal energy distribution for a fixed number of layers $N$, when $P\left(a_{i}\right)$ is concave.

Function construction of $P\left(a_{i}\right)$ using analytical models for signal strength and population distributions (Uniforms and Rayleigh) or from grid data generated by more advanced signal strength models and population databases, has been introduced. A related example for a transmitter located in Luleå where grid data for signal strength estimates and population were available was presented demonstrating significant system performance increases.

The methods presented in this paper show that physical layer optimization of multiresolution broadcast systems provide performance enhancements. Maximization of the value $\times$ population objective (and thereby possible revenue) provides best return on invested resources. Furthermore this provides a valuable tool in broadcast network planning to allow flexible rollout of new or tiered services.

\section{ACKNOWLEDGMENT}

The authors would like to thank Tor-Björn Minde Ericsson Research $\mathrm{AB}$ in Luleå for making the signal strength and population data available and Mats Ek of Progira Radio Communication $\mathrm{AB}$ in Luleå for signal strength calculations and help with data.

\section{REFERENCES}

[1] ETSI EN 300 744, "Digital video broadcasting (DVB); framing structure, channel coding and modulation for digital terrestrial television,", June 2004.

[2] D. Anastassiou, "Digital television," Proceedings of the IEEE, vol. 82 no. 4, pp. 510-519, April 1994.

[3] M. Sablatash, "Transmission of all-digital advanced television: state of the art and future directions," IEEE Transactions on Broadcasting, vol. 40, no. 2, pp. 102-121, June 1994.

[4] T. Cover, "Broadcast channel," IEEE Transactions on Information Theory, vol. IT-18, no. 1, pp. 2-14, January 1972. 
[5] K. Ramchandran, A. Ortega, M. Uz, and M. Verrerli, "Multiresolution broadcast for digital HDTV using joint source/channel coding," IEEE Journal on Selected Areas in Communication, vol. 11, no. 1, pp. 6-23, January 1993.

[6] W. Schreiber, "Spread-spectrum television broadcasting," SMPTE Journal, pp. 538-549, August 1992.

[7] L. Yun and D. Messerschmitt, "Variable quality of service in CDMA systems by statistical power control," in Proceedings of IEEE International Conference on Communication, 1995.

[8] M. Morimoto, H. Harada, M. Okada, and S. Komaki, "A study on power assignment of hierarchical modulation schemes for digital broadcasting," IEICE Transactions on Communications, vol. E77-B, no. 12, pp. 1495-1500, December 1994.

[9] S. Pradhan and K. Ramchandran, "Efficient layered data transport over multicarrier systems using optimized embedded modulation," IEEE Transaction on Communication, vol. 50, no. 6, pp. 877-881, June 2002.

[10] M. Vetterli and M. Uz, "Multiresolution coding techniques for digital television: a review," Multidimensional Systems and Signal Processing, no. 3, pp. 161-187, 1992.

[11] ITU-T Recommendation H.264, "Advanced Video Coding for Generic Audiovisual Services,", 2003.

[12] J. Ostermann, J. Bormans, P. List, D. Marpe, M. Norrschke, F. Pereira, T. Stockhammer, and T. Wedi, "Video coding with H.264/AVC: tools, performance, and complexity," IEEE Circuits and Systems Magazine, vol. 4, no. 1, pp. 7-28, First Quarter 2004.

[13] T. Wiegand, G. Sullivan, G. Bjøtegaard, and K. A, "Overview of the H.264/AVC video coding standard," IEEE Transactions on Circuits and Systems for Video Technology, vol. 13, no. 7, pp. 560-576, July 2003.

[14] H. Hamazumi, Y. Ito, and H. Miyazawa, "Adaptively weighted code division multiplexing for hierarchical digital broadcasting," NHK Laboratories Note, no. 437, pp. 1-13, September 1995.

[15] M. T. Heath, Scientific Computing: An Introductory Survey: McGraw Hill, 1997.

[16] S. G. Nash and A. Sofer, Linear and Nonlinear Programming: McGraw Hill, 1996.

[17] J. C. Spall, Introduction to Stochastic Search and Optimization: WileyInterscience, 2003.

[18] M. Hata, "Empirical formula for propagation loss in land mobile radio services," IEEE Transaction on Vehicular Technology, vol. VT-29, no. 3, pp. 317-325, August 1980.

[19] IEEE Vehicular Technology Society committee on radio propagation, "Coverage prediction for mobile radio systems operating in the 800/900 $\mathrm{MHz}$ frequency range," IEEE Transactions on Vehicular Technology, vol. 37, no. 1, pp. 3-72, February 1988.

[20] ITU-R Recommendation P.1546-1, "Method for Point to Area Predictions for Terrestrial Services in the Frequency Range $30 \mathrm{MHz}$ to 3000 MHz,", April 2003.
[21] E. W. Weisstein, CRC Concise Encyclopedia of Mathematics: Chapman \& Hall/CRC, 1999.

[22] N. G. de Bruijn, Asymptotic Methods in Analysis. Amsterdam, London: North-Holland Publishing Company, 1970.

[23] B. Barber, D. Dobkin, and H. Huhdanpaa, "The quickhull algorithm for convex hulls," ACM Transactions on Mathematical Software, vol. 22, no. 4, pp. 469-483, December 1996.

[24] Progira Radio Communication AB. (2004) GiraPlan 2.1-DVB-T and DAB Network and Planing Software. [Online] Available: www.progira.com

[25] Communications Research Centre Canada. (2004) CRC-Predict 3.21-Signal Propagation Model. [Online] Available:www.crc.ca

[26] Statistics Sweden (SCB). (2004) Population Statistics for Sweden. [Online] Available: www.scb.se

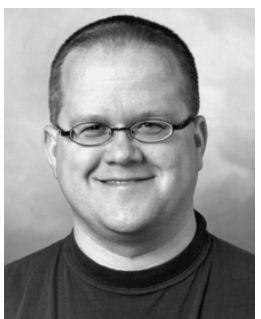

Martin Sehlstedt (S'01) was born in Kålaboda, Sweden, in 1966. He received the M.S. degree in computer science from Luleå University of Technology, Luleå, Sweden, in 2000.

He is currently Ph.D. student at the Division of Systems and Interaction, Luleå University of Technology. His primary research interests are statistical signal processing, optimization, and digital communication, with emphasis on wireless systems.

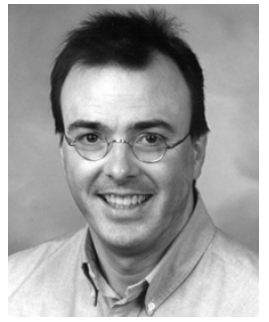

James P. LeBlanc (S'91-M'95-SM'98) was born in Kenmore, NY, in 1961. He received the B.S. degree from Rensselaer Polytechnic Institute, Troy, NY, in 1983, and the Ph.D. degree from Cornell University, Ithaca, NY, in 1995, both in electrical engineering.

$\mathrm{He}$ worked in industry for several years and was a member of the academic staff of New Mexico State University. In 2000, he joined Luleå University of Technology, Luleå, Sweden, where as of June 2004 he is full professor in signal processing. His interests are statistical signal processing, blind equalization/identification, and systems theory. 